\title{
Manejo Quirurgico del Torax Inestable: Experiencia en la Fundación Valle del Lili
}

\author{
Andrés Fernando Domínguez Calero, Mauricio Velásquez, Carlos A Ordoñez D
}

\begin{abstract}
O
El tórax inestable es una entidad muy compleja con una gran morbi-mortalidad asociada, que tradicionalmente había sido de manejo medico. Presentar la experiencia del manejo quirúrgico del tórax inestable en la Fundación Valle del Lili en el 2011 y los buenos resultados que se han tenido con este. Durante el período de tiempo comprendido entre enero del 2011 a mayo de 2012 se realizó un estudio descriptivo, prospectivo para evaluar los desenlaces de los pacientes con tórax inestable que se presentaron en la unidad de urgencias de la Fundación Valle del Lili. Se incluyeron todos los pacientes mayores de 18 años que tuvieran un traumatismo cerrado o abierto de la pared del tórax, con dos o mas fracturas costales. El material de osteosíntesis usado en todos los pacientes fue STRATOS ${ }^{\circledR}$ de la casa MEDXPERT. Se operaron 10 pacientes, (dos mujeres) con edad promedio de 55 (33-68) años. El tiempo quirúrgico promedio fue de 132 minutos. Solo 5 pacientes requirieron intubación prequirúrgica, y todos se extubaron en las 48 horas del postoperatorio. Estancia hospitalaria promedio de 10 días, la mejoría del dolor ocurrió en promedio en el día 3 postoperatorio excluyendo un paciente que por su estado neurológico no fue posible determinar ciertas variables; este fue el único que requirió traqueostomía. Hubo dos complicaciones respiratorias: Neumonía y oxígeno en casa. El tubo de tórax se dejó en promedio 5 días. Ningún paciente falleció como consecuencia del traumatismo ni de la cirugía. En este trabajo se muestra la experiencia inicial con el manejo quirúrgico evidenciando resultados favorables pero se requieren estudios con más poder para generalizar los resultados.
\end{abstract}

Palabras claves: Tórax inestable, Fijación costal, Trauma de tórax, Cuidado intensivo.

\section{ABSTRACT}

Flail chest is a complex entity which have been treated with medical support, with high rate of morbidity and mortality. Our goal is to examine the outcomes on surgical management of flail chest in Fundacion Valle del LILI, From January to May 2012, a case series study was conducted to evaluate the outcomes of patients with flail chest. The inclusion criteria included patients over the age of 18 years old who suffered a chest close trauma. Included different variables were demographics, hospitalization length, ICU length of stay, number of ribs fractured, duration under mechanical ventilation and complications. in all patients osteosynthesis material was used as STRATOS ${ }^{\circledR}$ from MEDXPERT. 10 patients were operated, avarage age of 55 years old (33-68). Avarage surgical time was 132 minutes. Five patients required presurgical intubation, all were extutbated in the next 48 hours postoperation. Average hospitalization length 10 days, average pain control at third day postoperative. One of the patients developed pneumonia and a patient required home oxygen. Chest tube was in for an average of 5 days. No death as a concequence of the trauma or the surgery. In this case series we shown an initial experience in surgical management of the flail thorax, our experience showed favorable outcomes regarding hospitalization length, postoperative pain control and mortality, Larger studies are needed to extrapolate to the general population.

Keywords: Flail chest, Costal fixation, Thoracic trauma, Intensive care.

How to cite this article: Calero AFD, Velásquez $M$, Ordoñez DCA. Manejo Quirurgico del Torax Inestable: Experiencia en la Fundación Valle del Lili. Panam J Trauma Critical Care Emerg Surg 2012;1(3):215-218.

\section{Source of support: Nil}

Conflict of interest: None declared

\section{INTRODUCCIÓN}

Las lesiones torácicas son muy frecuentes en los pacientes politraumatizados y alcanzan a provocar un 20 to $25 \%$ de todas las muertes traumáticas cada año en los Estados Unidos. $^{8}$

El tórax inestable se presenta apenas en un 5\% aproximadamente de los pacientes con fracturas costales, sin embargo es responsable de una mortalidad hasta de un 30 to $40 \%$ con efectos a largo plazo, ${ }^{8}$ como alteración en las pruebas de función pulmonar, dolor torácico crónico y sensación de disnea, en un poco mas de la mitad de los pacientes. $^{9}$

Tradicionalmente el manejo del tórax inestable ha sido médico con ingreso a una unidad de cuidado intensivo y quedando el manejo quirúrgico relegado a casos muy seleccionados. ${ }^{4}$ Sin embargo nuevos estudios han demostrado que el manejo quirúrgico es fundamental para una recuperación mejor y más rápida, ${ }^{3,5,8}$ por lo que se decide presentar la experiencia de un centro en el manejo quirúrgico del tórax inestable, En este estudio se presenta la experiencia del manejo quirúrgico del tórax inestable en una institución de IV nivel de atención en Cali, Colombia.

\section{MATERIALES Y METODOS}

Este es un estudio prospectivo descriptivo de los pacientes manejados quirúrgicamente por tórax inestable en una institución de IV nivel que se realizó entre enero de 2011 y mayo de 2012.

Se incluyeron todos los pacientes mayores de 18 años que tuvieran un traumatismo cerrado o abierto de la pared del tórax, con dos o mas fracturas costales y que provocara

Paper presented at the resident competition, 25th Annual Congress of Panamerican Trauma Society, Medellin, Colombia, November 2012. 
cualquiera de las siguientes situaciones para considerarlo como un tórax inestable:

- Falla en el destete de ventilación mecánica.

- Movimiento paradójico del tórax.

- Dolor severo o incapacitante para actividades simples como comer, orinar, toser, sentarse en el borde de la cama.

- Falla en las medidas analgésicas endovenosas, de anestesia regional o conductiva.

- Colapso de un hemitórax.

- Desplazamiento costal severo.

- Evidencia de penetrancia de las fracturas costales al tórax. Se excluyeron pacientes que por alguna razón hubieran sido llevados a toracotomía y durante el procedimiento se les hubieran fijado las fracturas costales con cualquier método.

Se registraron variables demográficas y otras de interés como el tiempo quirúrgico, estancia hospitalaria, estancia en UCI, presencia de lesiones asociadas, el número de costillas fracturadas, la necesidad y duración de ventilación mecánica invasiva y no invasiva, la duración del tubo de tórax, y la presencia de complicaciones relacionadas con el diagnostico y el procedimiento son descritas en esta serie.

Para el manejo quirúrgico se utilizo una técnica quirúrgica con un abordaje planeado según el sitio de las fracturas, haciendo incisiones limitadas en la pared del tórax y tratando de preservar la musculatura extrínseca del tórax como se muestra en la Figura 1, se utilizó el material de osteosíntesis costal de la casa Alemana MedXpert $\mathrm{GmbH}^{\circledR}$ conocido como sistema STRATOS ${ }^{\circledR}$.

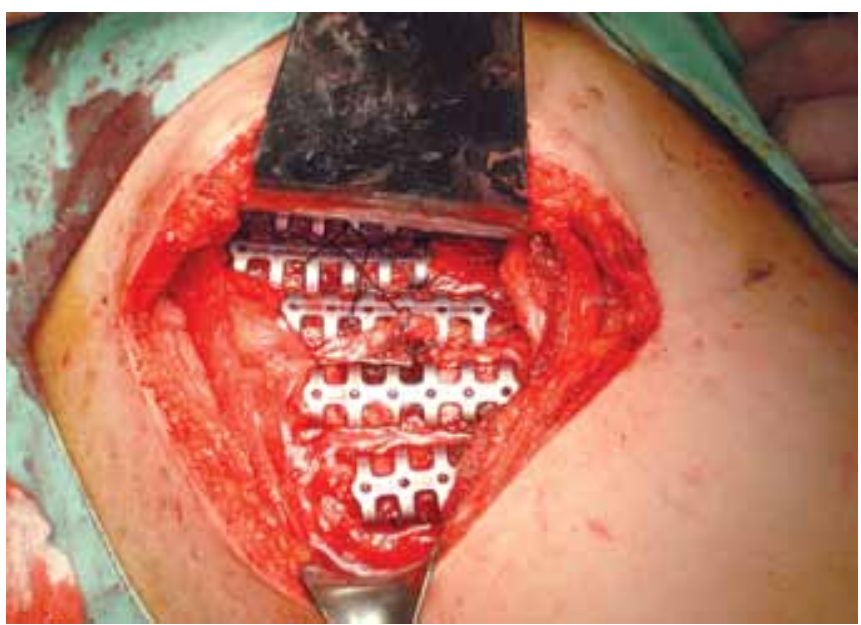

Figura 1: Paciente con reducción de fracturas costales y sistema de osteosíntesis posicionado, sistema STRATOS ${ }^{\circledR}$

\section{RESULTADOS}

Se operaron 10 pacientes durante el período de tiempo del estudio, de estos tres mujeres las dos primeras con un promedio de edad de 55 (33-68) años y la otra de edad mas joven de 22 años.

La indicación de cirugía en todos los pacientes fue la presencia de dolor severo que no mejoraba con el manejo analgésico adecuado y uno de estos pacientes presento movimiento paradójico del tórax debido a una desinserción de la pared torácica del esternón en el lado izquierdo con fractura esternal.

\begin{tabular}{|c|c|c|c|c|c|c|c|}
\hline Caso & Edad & Genero & Lesiones a sociadas & $\begin{array}{l}\text { \#Costillas } \\
\text { fracturadas }\end{array}$ & $\begin{array}{l}\text { Intubacion } \\
\text { preqx }\end{array}$ & $\begin{array}{l}\text { Tiempo } \\
Q x^{\delta}\end{array}$ & $\begin{array}{l}\text { Extubacion } \\
\text { postq }^{\varphi}\end{array}$ \\
\hline 1 & 51 & M & Hemotórax, contusión pulmonar & 4 & No & 120 & \\
\hline 2 & 67 & M & $\begin{array}{l}\text { Hemoneumotorax, fractura } \\
\text { humeral }\end{array}$ & $\begin{array}{l}8 / 4 \text { EN } 2 \\
\text { PUNTOS }\end{array}$ & No & 87 & \\
\hline 3 & 54 & $\mathrm{~F}$ & Confusión pulmonar & 5 & No & 108 & \\
\hline 4 & 63 & $\mathrm{~F}$ & Hemotorax, trauma hepatico & $\begin{array}{c}\text { 6/4 EN } 2 \text { O MAS } \\
\text { PUNTOS }\end{array}$ & No & 240 & \\
\hline 5 & 68 & M & Fractura esternal & $\begin{array}{l}3 / 2 \text { EN } 2 \\
\text { PUNTOS }\end{array}$ & $\mathrm{Si}$ & 35 & 3 \\
\hline 6 & 33 & M & $\begin{array}{l}\text { Hemoneumtorax, leceración } \\
\text { pulmonar }\end{array}$ & 3 & Si & 133 & 2 \\
\hline 7 & 63 & M & Hemotórax, contusión pulmonar & $\begin{array}{l}11 / 9 \text { EN } 2 \text { O } \\
\text { MAS PUNTOS }\end{array}$ & $\mathrm{Si}$ & 125 & 1 \\
\hline 8 & 42 & M & Hemoneumotórax & 4 & No & 210 & \\
\hline 9 & 57 & M & $\begin{array}{l}\text { Atelectasia lóbulo inferior } \\
\text { izquierdo, fractura conminuta de } \\
\text { escápula }\end{array}$ & 6 & $\mathrm{Si}$ & 80 & 1 \\
\hline 10 & 22 & $F$ & $\begin{array}{l}\text { Hemoneumotórax izquierdo, } \\
\text { confusión pulmonar, trauma } \\
\text { esplénico, fractura de cadera }\end{array}$ & 9 & $\mathrm{Si}$ & 135 & 5 \\
\hline
\end{tabular}

$\beta$ : Prequirúrgico; $\delta:$ Quirúrgico en minutos; $\varphi$ : Postquirúrgico en días 
Manejo Quirurgico del Torax Inestable: Experiencia en la Fundación Valle del Lili

\begin{tabular}{|c|c|c|c|c|c|}
\hline Caso & $\begin{array}{c}\text { Estancia } \\
\text { hospitalaria }^{\varphi}\end{array}$ & Majoria del dolor ${ }^{\alpha}$ & $\begin{array}{c}\text { Complicaciones } \\
\text { respiratorias }\end{array}$ & $\begin{array}{c}\text { Duracion } \\
\text { toracostomia }\end{array}$ & $\begin{array}{c}\text { Choque y } \\
\text { vasoactivos }\end{array}$ \\
\hline 1 & 9 & 2 & No & 4 & No \\
\hline 2 & 9 & 4 & No & 8 & No \\
\hline 3 & 9 & 2 & No & 5 & No \\
\hline 4 & 10 & 4 & $\mathrm{Si}$ & 3 & No \\
\hline 5 & 26 & SD & $\mathrm{Si}$ & 13 & $\mathrm{Si}$ \\
\hline 6 & 8 & 7 & No & 2 & $\mathrm{Si}$ \\
\hline 7 & 8 & 2 & No & 3 & $\mathrm{Si}$ \\
\hline 8 & 5 & 2 & No & 2 & No \\
\hline 9 & 4 & 4 & No & NR & No \\
\hline 10 & 23 & 4 & No & 4 & No \\
\hline
\end{tabular}

$\Phi:$ Estancia en días; $\alpha$ : Día postoperatorio en el que hubo mejoría del dolor; $\Psi$ : Duración en días; SD: Sin Dato

En la Tabla 1 se pueden apreciar los datos preoperatorios; 5 de nuestros pacientes necesitaron intubarse antes de la cirugía, el tiempo promedio de cirugía fue de 132 minutos y los cinco pacientes se pudieron extubar en el postoperatorio en promedio al segundo día.

En la Tabla 2 se pueden apreciar los resultados del manejo dado a los pacientes, se tuvo una estancia hospitalaria de 10 días en promedio, la mejoría del dolor ocurrió en promedio en el día 3 postoperatorio excluyendo un paciente (caso 5) que presento una enfermedad cerebral isquémica secundaria a un embolismo cerebral debido a una arritmia cardíaca por el trauma cerrado del corazón, que por su estado neurológico no fue posible determinar ciertas variables y fue el único que requirió traqueostomía. Solo hubo dos complicaciones respiratorias, que se presentaron en los casos 4 y 5 que tuvieron una neumonía y la necesidad de oxígeno en casa. El tubo de tórax se dejó en promedio 5 días. Los casos 5, 6 y 7 tuvieron un postoperatorio mas complejo con necesidad de vasoactivos que se pudo retirar en promedio en el día 2 postoperatorio.

Ningún paciente falleció como consecuencia del traumatismo y de la cirugía.

\section{DISCUSION}

El traumatismo torácico es una entidad muy frecuente entre los pacientes politraumatizados ${ }^{1,2}$ y aunque el tórax inestable se presenta en apenas un 5\% de los pacientes con trauma torácico, este es responsable de un 30 a $40 \%$ de la mortalidad por el traumatismo del tórax. ${ }^{1,4}$

El manejo médico había sido una de las opciones terapéuticas mas aceptadas dejando el manejo quirúrgico para algunos casos muy seleccionados, ${ }^{3,6}$ sin embargo las complicaciones asociadas al manejo médico como intubación prolongada, neumonía, estancias prolongadas en cuidado intensivo y de hospitalización al igual que las complicaciones a largo plazo de las lesiones producidas por el traumatismo torácico (dolor crónico, incapacidad de laborar tiempo completo, deformidades en el tórax y dificultad respiratoria) ${ }^{4,6,11}$ y el desarrollo de nuevos materiales y de la técnica quirúrgica han hecho resurgir la alternativa de la cirugía como parte integral del manejo del tórax inestable. ${ }^{2,3,5}$

En esta serie de casos identificamos que una estabilización quirúrgica temprana tiene ventajas y mejores resultados tal como lo muestra el trabajo de Tanaka y colaboradores, ${ }^{3}$ disminuyendo la necesidad de ventilación mecánica, un control analgésico mas fácil y menos complicaciones derivadas del manejo medico como son sensación de disnea, infecciones, alteración de las pruebas de función pulmonar, dolor torácico crónico y reincorporación a actividades laborales de forma más temprana; en nuestra serie de casos se encontró que se han manejado casos muy complejos por una gran afección en la pared torácica como los casos $2,4,5,7$ y 10 y el tratamiento ofrecido mostro resultados favorables en relación a la estancia hospitalaria menor requerimiento de cuidado intensivo, la cual fue muy inferior a la reportada en los diferentes trabajos reportados en la literatura. ${ }^{3,4,7,8,10}$

Esta serie de casos muestra la utilidad del uso de grapas de titanio el cual tiene una compatibilidad con los tejidos extraordinaria y resiste a la corrosión por contacto con el aire y el entorno biológico, ${ }^{12}$ para la fijación costal del tórax inestable, la reconstrucción de defectos traumáticos de la pared torácica y la reducción y fijación quirúrgica de fracturas costales desplazadas, que sean móviles y dolorosas o con deformidades torácicas tales que no impliquen dos fracturas en una misma costilla pero que si sean tantas o tan desplazadas como para generar una inestabilidad torácica. ${ }^{7}$

Aunque la estabilización quirúrgica de las fracturas costales no puede recomendarse para todos los pacientes, en 
este momento, como una técnica generalizada, en un grupo selecto de pacientes con lesiones de la pared torácica su uso puede mejorar la evolución. ${ }^{3-5}$

La mejoría del dolor es notable y en promedio se logró en el 2 día postoperatorio, lo cual unido a un retiro del tubo de tórax en promedio en el día 5 postoperatorio permitió una deambulación mas temprana y la disminución en los días de estancia hospitalaria lo cual esta en relación a la serie de pacientes reportada en España por Moreno De La Santa Barajas y colaboradores. $^{5}$

Solamente un paciente de 10 presento una neumonía y es un caso bastante complejo por la complicación neurológica del traumatismo sufrido y una ventilación mecánica mas prolongada.

La extubación del caso de la paciente 10 fue un poco mayor secundario a la contusión pulmonar asociada al trauma de esta paciente.

\section{CONCLUSIONES}

El tórax inestable es una entidad muy compleja con una gran morbimortalidad asociada que tradicionalmente había sido de manejo medico. En este trabajo se muestra la experiencia inicial con el manejo quirúrgico demostrando unos resultados bastante positivos, sin embargo se requieren de mas estudios con un mayor numero de pacientes que permitan generalizar los resultados y afinar mas las indicaciones de manejo quirúrgico del tórax inestable.

\section{REFERENCIAS}

1. Nirula R. Rib Fracture repair: Indications, technical issues, and future directions, World J Surg 2009;33:14-22.

2. Tagawa T, Itoh S, Ide S, Tanaka K, Yoshida K, Ohe H. Repair of intrathoracic visceral damage using video-assisted thoracoscopic surgery for blunt chest trauma and rib fixation at the site of minithoracotomy. Jpn J Thorac Cardiovasc Surg 1998;46:121-26.

3. Tanaka H, Yukioka T, Yamaguti Y, et al. Surgical stabilization of internal pneumatic stabilization? A prospective randomized study of management of severe flail chest patients. J Trauma 2002;52:727-32.

4. Granetzny A, Abd El-Aal M, Emam E, Shalaby A, Boseila A Surgical versus conservative treatment of flail chest Evaluation of the pulmonary status. Interact Cardiovasc Thorac Surg 2005;4:583-87.

5. De La Santa Barajas PM. Fijación quirúrgica de las fracturas costales con grapas y barras de titanio (sistemaSTRATOS). Experiencia preliminar, CIR ESP 2010;88(3):180-86.

6. Ahmed Z, Mohyuddin Z. Management of flail chest injury: internal fixation versus endotracheal intubation and ventilation. J Thorac Cardiovasc Surg 1995;110:1676-80.

7. Easter A. Management of patients with multiple rib fractures. Am J Crit Care 2001;10:320-27, quiz 8-9.

8. Kiraly L, Schreiber M. Management of the crushed chest. Crit Care Med 2010;38(9) (Suppl).

9. Derdak S, Mehta S, Stewart TE, et al. High frequency oscillatory ventilation for acute respiratory distress syndrome in adults: A randomized, controlled trial. Am J Respir Crit Care Med 2002; 166:786-87.

10. Lardinois D, Krueger T, Dusmet M. Pulmonary function testing after operative stabilisation of the chest wall for flail chest. Eur J Cardiothorac Surg 2001;20:496-501.

11. Athanassiadi K, Gerazounis M, Theakos N. Management of 150 flail chest injuries: Analysis of risk factors affecting outcome. Eur J Cardiothorac Surg 2004;26:373-76.

12. Hauser J, Koeller M, Bensch S, Halfmann H, Awakowicz P, Steinau HU, et al. Plasma mediated collagen-I-coating of metal implant materials to improbe' biocompatibility. J Biomed Mater Res A 2010;20.

\section{ACERCA DE LOS AUTORES}

\section{Andrés Fernando Domínguez Calero (Correspondiente Autor)}

Residente de Cirugía General, Universidad CES, Cali, Colombia e-mail: andrewmedico@hotmail.com

\section{Mauricio Velásquez}

Cirujano General, Cirujano de Tórax, Docente Universidad Ces, Cali Colombia

\section{Carlos A Ordoñez D}

Cirujano General, Cirujano de Trauma, Emergencias y Cuidado Intensivo; Profesor Titular de Cirugía General, Trauma y Cuidado Intensivo Universidad del Valle, Fundación Valle del Lili, Director y Jefe de Sección de Cirugía de Cirugía de Trauma y Emergencias Universidad del Valle, Cali, Colombia 